\title{
COVID-19 as Information Transmitter to Global Equity Markets: Evidence from CEEMDAN-Based Transfer Entropy Approach
}

\author{
Peterson Owusu Junior $\mathbb{D}^{1},{ }^{1}$ Siaw Frimpong $\mathbb{D}^{1},{ }^{1}$ Anokye M. Adam ${ }^{D},{ }^{1}$ Samuel K. Agyei $\left(\mathbb{D},{ }^{1}\right.$ \\ Emmanuel N. Gyamfi $\mathbb{D}^{2},{ }^{2}$ Daniel Agyapong $\mathbb{D}$, ${ }^{1}$ and George Tweneboah $\mathbb{D}^{3}$ \\ ${ }^{1}$ Department of Finance, School of Business, University of Cape Coast, Cape Coast, Ghana \\ ${ }^{2}$ GIMPA Business School, Ghana Institute of Management and Public Administration, Accra, Ghana \\ ${ }^{3}$ Wits Business School, University of the Witwatersrand, Johannesburg, Parktown, South Africa \\ Correspondence should be addressed to Anokye M. Adam; aadam@ucc.edu.gh
}

Received 14 July 2021; Revised 1 August 2021; Accepted 13 August 2021; Published 27 August 2021

Academic Editor: Yuxing Li

Copyright ( 92021 Peterson Owusu Junior et al. This is an open access article distributed under the Creative Commons Attribution License, which permits unrestricted use, distribution, and reproduction in any medium, provided the original work is properly cited.

\begin{abstract}
This study provides an analysis of chaotic information transmission from the COVID-19 pandemic to global equity markets in a novel denoised frequency domain entropy framework. The current length of the pandemic data offers the opportunity to examine its role in the asymmetric behaviour patterns of investors according to time horizons and the diversification potentials available to them. We employ the total daily global confirmed cases of COVID-19 and 27 equity indices from December 31, 2019, to April 18, 2021. Our results corroborate the idea that diversification potentials are stronger in the short to medium term. The Global Index (higher risk) and Canada and New Zealand (lower risk) remain at both ends to pair some other equities to offer diversification prospects because of the transmission of information from COVID-19 to the selected equity markets. In addition, we provide the source of these diversification prospects as information flow rather than transmission of shocks, which is common in the literature. Furthermore, our results suggest detailed levels of risk (lower vis-à-vis higher) in the situation where they have been stripped of the noise in the market. The findings allow both investors and policymakers to make informed decisions based on the time horizons since the pandemic communicates different chaotic information with the lapse of time. This is imperative to avoid the negative consequences of the increasing infection rate on global stock markets.
\end{abstract}

\section{Introduction}

The outbreak of coronavirus disease 2019 (COVID-19) as an epidemic in Wuhan, China, took the world by surprise to become a global pandemic on March 11, 2020 (WHO, 2020). On April 18, 2020, the global confirmed cases of the pandemic had reached $141,404,998$ with more than 387,352 deaths per million [1]. While there are ongoing mass vaccinations in different parts of the world, the spread of the virus has not come to a stop (https://covid19.who.int/). Different variants keep popping up in different places. However, the rate of spread has changed from an increasing rate to a decreasing rate. This suggests hopes of containing and finally providing a cure in the long term. However, the pandemic is still here and the economic, social, and financial havoc it has wreaked so far is yet to be fully quantified. This has led to major disruptions in personal lives, businesses, and economic activities, with more than half of the world's population currently under restrictions of movement and partial lockdown $[2,3]$. The impact that "pausing" the economy may have on supply chains, household demand, and the financial stability of the economy is largely unknown [3].

Many governments across the globe have resorted to drastic measures such as enhanced quarantine processes, lockdown (total or partial), limiting human contacts through social distancing, and contact tracking and tracing through their cell phones to curb the rate of infection of COVID-19. As a panic result, a phenomenon due to COVID-19 fear erupted in all markets that have caused a shortage of food 
and everyday goods in supermarkets and a drop in share prices $[2,4]$. In the US, the stock market plunged by $12 \%$ amid COVID-19 fears against all 11 groups in the S\&P 500 falling on March 16, 2020. Share prices in Asia and Europe also fell, and the yield of bonds declined in most parts of Europe, where a measure of market stress hit levels not experienced since the euro crisis in 2011-2012. On April 6, 2020 , most stocks soared following a reduction in daily new cases over the previous weekend. The COVID-19 pandemic has proven its potency to be contagious with financial assets. This evidence corroborates the tenets of the efficient market theory, as it is known to its adherents. The hypothesis holds that the market prices of most stocks are correctly priced based on the information available at that time $[5,6]$. The theory posits that when markets are efficient, all available relevant information about a financial asset is utilised in fixing current prices to reflect the fact that the optimal forecast return is equivalent to the market equilibrium return. Impliedly, information that suggests future economic activity is a key conditioning factor in determining the current prices of financial assets. Thus, this paper argues that global financial markets are likely to respond to communications on "pausing" the economy and its implications, such as a reduction in productive activities, an increase in unemployment, a gradual reduction in market participants, and the general uncertainty primarily caused by COVID-19. Based on the prediction of the efficient market theory, market prices are expected to dip under the current global conditions created by COVID-19. But the evidence against the theory such as the January effect, market overreaction, excessive volatility, mean reversion, and delayed effect of price responses to information $[7,8]$ and the fact that global financial market efficiency levels are not homogeneous place the empirical investigation in perspective.

Nonetheless, the behavioural patterns of investors tend to differ across time, especially under turbulent times such as those we are experiencing now. This asymmetric and timebased behaviour of investors is what reflect in the market prices because the market does not operate in a vacuum. Two theories that support this phenomenon are the adaptive market hypothesis (AMH) [9] and the heterogeneous market hypothesis (HMH) [10]. While the EMH assumes that the rationality of investors depends on neither time nor circumstances [11], Cornell [11] argues that rationality (and irrationality) is state-dependent over time and between investors. Thus, in "What is the Alternative to Market Efficiency" (AHME), Cornell [11] captures both AMH and $\mathrm{HMH}$ in the sense that the AMH enjoins the study of investor behaviour over small subsamples against the full sample. The latter reveal different levels of efficiency unlike the stationary and perpetually equilibrium notion of the EMH. Furthermore, the HMH advance that different economic agent takes their investment decisions on different time horizons in line with their risk and return preferences by analysing past and current news. To account for time horizon, the authors redefine time as intrinsic time which corresponds to time scales of short, medium, and long term. It becomes obvious that these behavioural dynamics introduce asymmetry, nonlinearity, nonstationary, and noise in the price-generating process of asset returns. Hence, empirical studies require methods that can account for all these complexities.

Theoretically and empirically, it is believed that these investor-generated complexities span across different markets that offer different levels, returns, and risks, in line with the generic idea of portfolio diversification. These markets are not only different, but their distinguishing feature is the competitive risks and returns they provide. Again, the market can be in the same asset class or not. Notably, competing risks and rewards seem to come from nontraditional asset classes such as cryptocurrencies, where traditional investors diversify into. Particularly, in turbulent market times, the assets and/or asset classes that competitively satisfy the investor's wishes are called safe havens [12]. Since the emergence of cryptocurrency as a nontraditional asset class, recent episodes of financial crises, and currently the COVID-19 pandemic, a plethora of studies have revisited the basic premise of portfolio diversification in search of competitive returns and risk levels [13-27]. The underlying thread in these and many studies is the idea that investors are always scouring competing risks and rewards and this search intensifies under stressed market conditions. Also, while information flows between markets precedents on investors' search, the flow deepens as well under difficult market periods. From the foregoing discourse, we sum this up and refer to it as the competitive market hypothesis $(\mathrm{CMH})$, which implies that, in part, the intensity of information flows and spillover between markets of the same and differing asset classes are exacerbated by rational, albeit irrational investors' relentless search of competing rewards and risks to satisfy the portfolio goals.

Generally, there is a paucity of literature on the relationship between pandemics and financial market behaviour. The tumbling of the various stock markets around the world offers the opportunity to understand their relationship with COVID-19. The relationship between pandemics and financial market prices could be direct through the related information dissemination channel and indirect through the pandemic-led weakened economic activity in the market because of a general reduction in income levels of market participants. Generally, empirical evidence suggests that the health conditions of economies help explain differences in wealth of nations [28-30] probably due to the loss of adequate and sufficient labour force. Barret et al. [31] argue that the cost of a pandemic goes beyond the loss of productivity due to sickness to include the cost of disease avoidance, caring for dependents, closure of schools, and the cost of dealing with behavioural changes that are likely to have economic outcomes.

The cost of COVID-19 is likely to also include loss of tax revenue, idle labour costs, increased social welfare cost, security cost, financial bailouts, damaged international relations, political mistrust, and hampering global efforts towards the achievement of sustainable development goals. Sander et al. [32] argue that pandemics such as influenza reduce per capita growth, but the extent of economic shrinkage depends on the mitigation measures put in place. Schwartz [33] corroborates this finding by predicting that a 
deep coronavirus-induced recession in the US is virtually inevitable. Notwithstanding, governments are likely to reduce operating costs by functioning at close to half capacity, even though this is not likely to be significant. Projected reductions in per capita income can influence financial market activity through the indirect channel of reducing the economic well-being of market participants. Unfortunately, however, there are few existing empirical studies on the economic implications of COVID-19, whose end is shrouded in uncertainty. Policy decisions are likely to be less informed because according to Reinhart [34], historical episodes are nonexisting. The consequence may be the creation of another global problem because of the desperate measures to solve one. The thrust of this paper is to provide a baseline study to support the formulation of global policies in these extraordinary times. The paper extends the scope of Schoenfeld [35] who evaluated the effect of COVID-19 on markets in the United States to paint a global picture of the effect of the pandemic on global equity markets.

Since COVID-19 was declared a pandemic, many studies have trickled in what one may call a "mad rush" to publish. These early studies have attempted to examine the impact of the pandemic on many aspects of our lives. Nonetheless, given the span of data during those earlier stages, it was not feasible to tell a good story which naturally evolved over time. The news and reality of the pandemic triggered panic in the early stages but had abated with time with the reduced infection rates and vaccinations. For more than a year, different investors have had ample time to rebalance their portfolios as they monitor the markets in their decisionmaking processes. This is enough time for the adaptive and heterogeneous behaviour of investors to become evident. This allows us to adequately track the dynamic relations resulting from the pandemic through the time horizons as evidenced by frequency decomposition (also referred to as frequency domain). Many early studies bear the limitation of not being able to show these dynamics. Furthermore, others attempted to examine the relationship between COVID-19 and equities using wavelet coherence [36], between COVID19 and energy commodities [37], and between financial and nonfinancial firms in China and G7 countries [38] with dynamic conditional correlation (DCC). Given the dataintensive nature of these techniques, the results from these studies can be perceived with caution. We opine that dataintensive studies with data spanning less than a year since the World Health Organisation (WHO) declared that the COVID-19 global pandemic may be a too small a sample. This is informed by a minimum forecasting length of one year as prescribed by the Bank for International Settlements (BIS) (https://www.bis.org/statistics/gli.htm? $\mathrm{m}=6 \% 7 \mathrm{C} 333 \%$ 7C690).

On the other hand, many studies on the impact of COVID-19 on equities have focused mainly on the quantum of spillover between markets (see, e.g., [18, 39-45]). However, it is evident that, under the circumstances, the driving force of these transmissions is panic or fear from the pandemic. Thus, it is more prudent to quantify the information flow between the markets using the right technique. For two or more variables evolving in time, a theoretic information measure can be quantified in terms of the driving and responding transfer. This is the transfer entropy as predicted by Schreiber [46]. Rightfully, a growing number of studies have employed the transfer entropy in investigating the impact of COVID-19. Notable among these is the study by Lahmiri and Bekiros [47] who used the largest Lyapunov exponent (LLE) based on the Rosenstein method and approximate entropy. This study is motivated, in part, by the robustness of the method to small samples. However, the sample at this stage is no longer small. Regarding studies that have looked at the frequency domain impact of COVID-19 on equity markets under information transfer, the literature is booming. Lahmiri and Bekiros [47] examined the impact of COVID-19 on the randomness of global equity volatilities based on the Shannon entropy wavelet transform domain. Wang and Wang [48] also used multiscale transfer entropy to examine the effect of COVID-19 on major global equity, currencies, and Bitcoin. These studies suffer from small sample bias, on the one hand, and the use of Shannon entropy, on the other hand. As it will be clear soon, the Shannon entropy is weak in assigning equal weights to different portions during the distribution of the data (see also $[49,50])$.

Our study is one of the few which fills these gaps identified in the literature. To do this, we employ the Complete Ensemble Empirical Mode Decomposition with Adaptive Noise- (CEEMDAN-) based entropy to quantify the information flow from COVID-19 to major global equities. In so doing, we use a longer sample period, which is needed to better understand the dynamics of investor behaviour. The CEEMDAN technique decomposes the time series into intramode decomposition (IMF) which represents different time horizons. This addresses the asymmetric, nonlinearity, and nonstationarity dynamics as espoused by the $\mathrm{AMH}$ and $\mathrm{HMH}$. Another strong feature of the CEEMDAN is that it can strip the noise that is embedded in the data due to the behaviour of irrational investors. This is also supported by the AHME as it encompasses both AMH and $\mathrm{HMH}$. Further, we employ a unique variant of entropy called Rényi entropy which can differentiate between the tails of the distribution by assigning appropriate weights. While it is well established that financial assets exhibit heavy tails, the Shannon entropy does not capture this. Particularly in a pandemic such as COVID-19, these fat tails are stronger and should be taken into account accordingly [51]. However, we provide results from the Shannon entropy for comparison purposes (see Figure 1 and Table 1).

Our study makes significant contributions to the nascent COVID-19 equity nexus. First, this is the first to employ noise-assisted techniques on equities to provide a clearer picture of the effect of the pandemic. It is shown that noise in signals (time series) can impact the results of the analysis to some extent that the noise can be more pronounced than the signal's effect [52]. The CEEMDAN reduces noise to its barest minimum. Second, the frequency decomposition itself offers a way to analyse the seemingly unwanted impact of the pandemic across time horizons. This allows investors and policymakers to tailor future decisions in line with the adaptive and heterogeneous nature of the market 

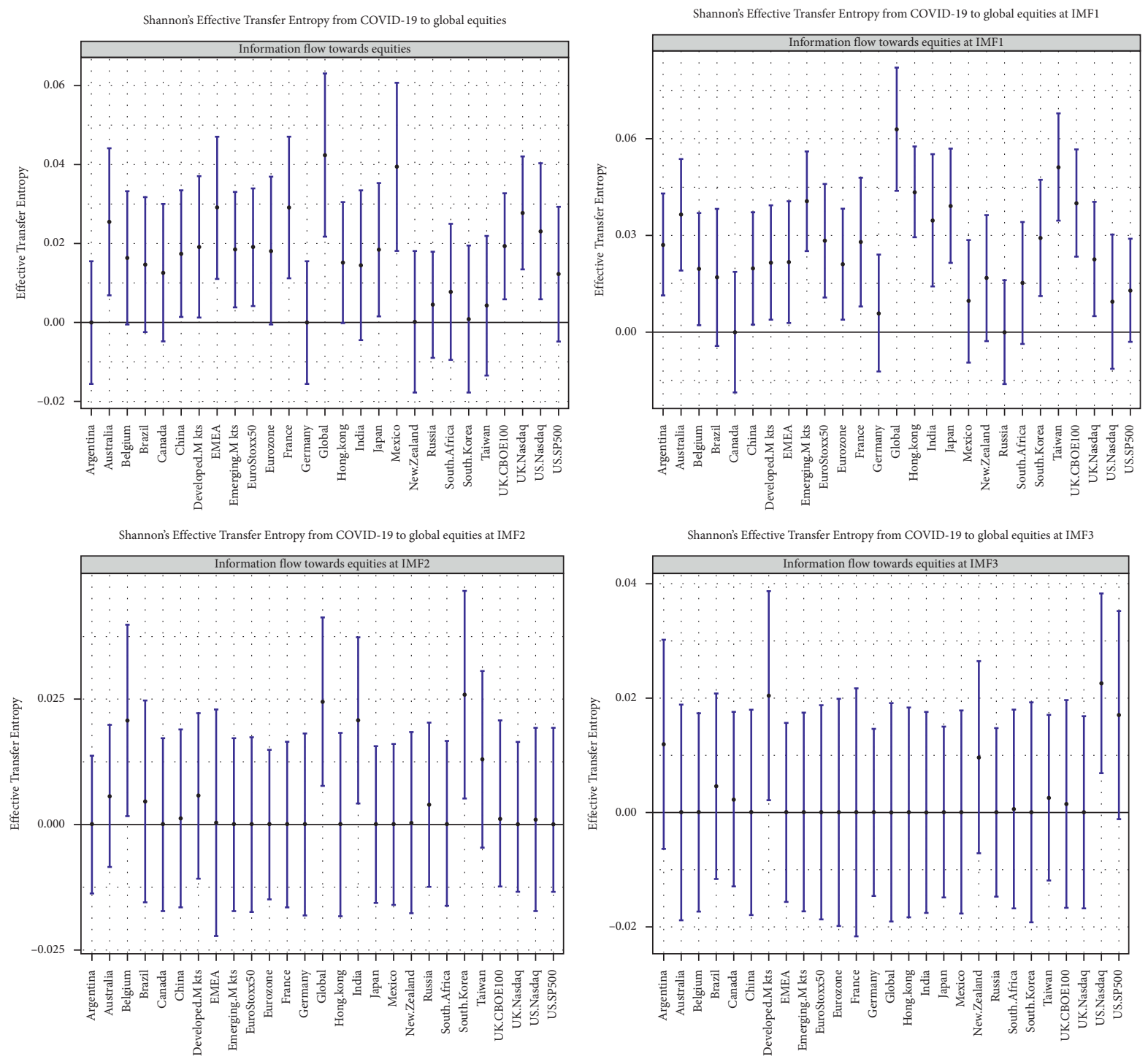

(a)

FIgURE 1: Continued. 

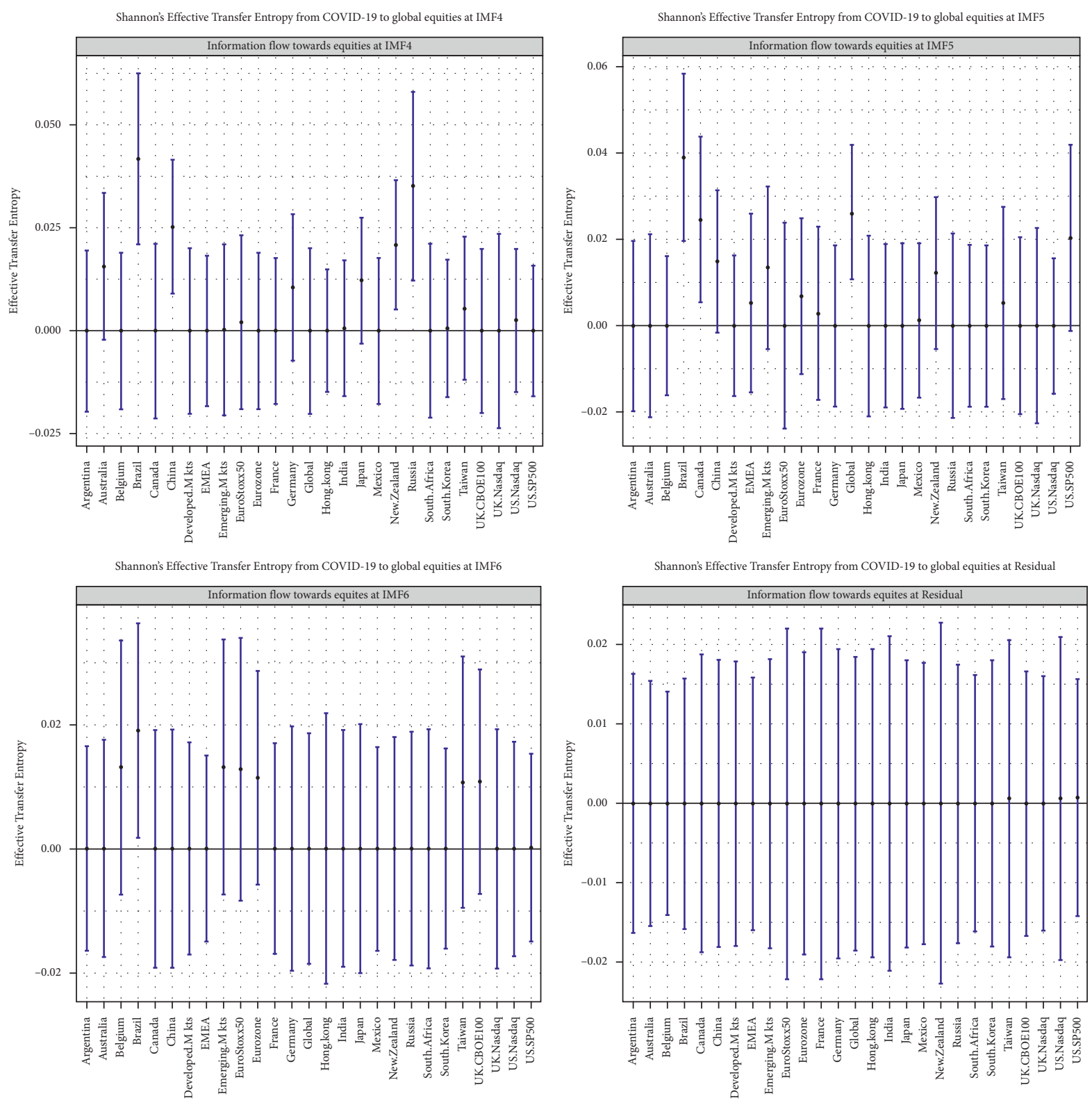

(b)

Figure 1: Shannon ETE from COVID-19 to global equity markets in composite and frequency domain.

TABLE 1: Summary effective transfer entropy from COVID-19 to global equities.

\begin{tabular}{|c|c|c|c|c|c|}
\hline \multirow{2}{*}{ Series } & \multirow{2}{*}{ Time horizon } & \multicolumn{2}{|r|}{ Rényi } & \multicolumn{2}{|c|}{ Shannon } \\
\hline & & Diversification potential & Equities involved & & \\
\hline Composite & - & Yes & $\begin{array}{l}\text { Global versus Australia, Belgium, Canada, EMEA, France, } \\
\text { Mexico, UK (CBOE100), and UK (Nasdaq) }\end{array}$ & No & - \\
\hline IMF1 & Short term & Yes & $\begin{array}{c}\text { Global versus Argentina, China, UK (CBOE100), } \\
\text { and UK (Nasdaq) }\end{array}$ & No & - \\
\hline IMF2 & Short term & No & - & No & - \\
\hline IMF3 & Short term & No & - & No & - \\
\hline IMF4 & Medium term & No & - & No & - \\
\hline IMF5 & Medium term & Yes & $\begin{array}{c}\text { (Canada and New Zealand) versus Argentina, Belgium, } \\
\text { EuroStoxx50, Germany, India, and Taiwan }\end{array}$ & No & - \\
\hline IMF6 & Medium term & No & - & No & - \\
\hline Residual & Long term & No & - & No & - \\
\hline
\end{tabular}


participants. In the same vein, we provide an asymmetric, nonlinear, nonstationary, and nonparametric lens to view the effect of the pandemic on equity. These circumvent a myriad of estimation limitations which otherwise are difficult to deal with. Third, we use the transfer entropy technique to provide evidence quantifying the information flow between the markets. This is in contravention to spillover indices such as Diebold and Yilmaz [53] and Baruník and Křehlík [54], which ignore the intrinsic information driving the market. Transfer entropy methods also implicitly advance causality. In this case, we provide knowledge of the causality levels of COVID-19 to the global equity market. Thus, our study offers a result on asymmetric, nonlinear, and nonparametric causality. The use of this rich causality information is important for both policy and investment.

Fourth, since we employ the Rényi transfer entropy [55], we provide COVID-19 impact results that are consistent with stylised financial returns. In this regard, readers are confident that the fat-tail and leverage effect of asset returns have been accounted for. This knowledge is indispensable to make the right investment decision on asset allocation and risk analysis. Furthermore, we use the Rényi entropy to estimate the effective transfer entropy (ETE) which delineates lower-risk assets from higher-risk assets. The ETE provides negative and positive estimates for higher risk and lower-risk assets, respectively. With this information, investors are confident in the combination of assets in their portfolio, which can help minimize risk and achieve high returns during this pandemic. One novel contribution of this study is that we can diversify based on quantified negative and positive transfer of information rather than just spillover, as is commonplace in the diversification literature. This is a new lens through which to view diversification in a distribution-free asymmetric nonlinear frequency domain framework.

Overall, our results show that negative recipients increase with time horizon (IMFs) while positive recipients reduce. At the composite level, the Global Index (higher risk) can be paired with Australia, Belgium, Canada, EMEA, France, Mexico, UK CBOE100, and UK (Nasdaq), which are lower-risk assets. We find more diversification potential in the short term for the Global Index (higher risk) paired with Argentina, China, the UK, CBOE100, and the UK (Nasdaq) (lower risk) indices. In the medium term, Canada and New Zealand (lower risk) can be combined with Argentina, Belgium, EuroStoxx50, Germany, India, and Taiwan (higher risk) equities.

The remainder of the paper is structured as follows. In Section 2, the methodologies and CEEMDAN-based Rényi transfer entropy are presented. In Section 3, information on the data used and preliminary outputs is presented. Analysis of the main results is contained in Section 4, and Section 5 concludes the study.

\section{Methodology}

Our methodology involves a two-stage approach. First, we decompose the equity indices into intrinsic mode functions
(IMFs) using the CEEMDAN technique. The IMFs denote intrinsic time that correspond to short, medium, and long terms. This allows us to determine the effects of COVID-19 in different decision-making time frames. Second, we estimate the effective transfer entropy using the Rényi entropy (RE) specification. The RE quantifies the information flow from COVID-19 to the equity markets by assigning much weight to the tail of the distributions in line with the extant literature. Together, these combined methods address asymmetry, nonlinearity, and nonstationarity in the series [56] in a nonparametric way. In this study, we have elected to examine the unidirectional flow of information from COVID-19 to the equity markets. It is logical to assume that there is no transfer of information in the reverse direction. We also decide to keep the COVID-19 series in its composite form without decomposition. This choice is premised on the fact that the COVID-19 pandemic is not economic agent that updates its decision across time horizons. However, investors adjust their decisions based on the trend exhibited by the pandemic in the number of confirmed cases.

\subsection{Complete Ensemble Empirical Mode Decomposition.} Ramsey and Lampart [57] correctly put it that economists have long known that the relationships between variables in terms of form, degree, and direction differ across time horizons. However, the tools to decompose economic time series into all orthogonal time-scale components have been lacking until now. Further, tools for dealing with noise that usually dominates financial time series in the short term are currently available $[57,58]$. A typical example is the CEEMDAN which is the latest of the empirical mode decomposition (EMD) sequel started by Huang [59]. The strength of mode decomposition is its efficiency, reconstruction accuracy, and minimization of the noise-to-signal ratio (SNR) in unstable state signals [60]. The CEEMDAN proposed by Torres et al. [61] comes out on top in these qualities compared to their predecessors. For instance, while the EEMD does a relatively good job in removing noise, it fails to fully recover the original signal with the sum of IMFs. This limitation does not allow the EEMD to determine the SNR. However, CEEMDAN has mechanisms to obtain the SNR by appending the white noise to the residual of previous iterations instead of to the original signal [62]. We use the CEEMDAN mainly due to its noise-removal strength, especially for this study period of panic in the financial markets caused by the COVID-19. We implement this with the libeemd package (in the $\mathrm{R}$ environment for statistical computing and graphics).

The CEEMDAN algorithm is summarised as follows, adapted from Liu et al. [63]:

(1) Add a Gaussian white noise $\omega_{k}(t)$ of different amplitudes to a signal $x(t)=s(t)+n(t)$, where $s(t)$ is the actual signal and $n(t)$ is the noise. This results in many new signals:

$$
x_{k}(t)=x(t)+\omega_{k}(t) .
$$


(2) Employ EMD technique to decompose (1) to obtain the first IMF.

(3) Estimate the average of this first IMF as

$$
\overline{\operatorname{IMF}_{1}(n)}=\frac{1}{K} \sum_{k=1}^{K} \operatorname{IMF}_{1 k}(t)
$$

(4) Calculate the remainder of signal components $C_{n}$ of the original signal as

$$
C_{n}= \begin{cases}x(t)-\mathrm{IMF}_{1}(n), & n=1 \\ C_{n-1}-\operatorname{IMF}_{1}(n), & n>1\end{cases}
$$

(5) The $\rho$-th component post-EMD of the signal is denoted by $E_{\rho}$. Subsequent components ( $L+1$ IMFs) can be obtained by

$$
\operatorname{IMF}_{(L+1)}(n)=\frac{1}{K} \sum_{k=1}^{K} E_{L}\left\{C_{L}(t)+\sigma_{L} E_{L}\left(\omega_{k}(t)\right)\right\} .
$$

(6) Last, the $n$ steady-state IMFs are reconstructed to produce the original signal as

$$
x(t)=C(t)+\sum_{m=1}^{M} \overline{\operatorname{IMF}_{m}}(t) .
$$

The total number of IMFs (and one residual) of a time series signal is given as $\log _{2} N$, where $N=T$ (number of observations). For further reading on the empirical mode decomposition sequel; see [64-66].

2.2. Rényi Transfer Entropy. Transfer entropy finds its root in the general information theory of Hartley [67]. Hartley's [67] theory measures information via an algorithm on all possible number of symbolic sequences that can occur in a specific probability distribution $[68,69]$. The current literature on transfer entropy is based on the mathematical theory of communication by Shannon [70] as a measure of uncertainty, a derivation of information theory.

For a probability distribution with diverse symbols of a given experiment $P_{j}$, each symbol's average information is specified as

$$
H=\sum_{j=1}^{n} P_{j} \log _{2}\left(\frac{1}{P_{j}}\right) \text { bits, }
$$

where $n$ denotes the number of distinct symbols with respect to the probabilities $p_{j}$ [67]. In the Shannon's [70] framework (hereafter referred to as Shannon entropy), for a discrete random variable $J$ with probability distribution $p(j)$, the average number of bits needed to optimally encode independent draws can be given as [68]

$$
H_{J}=-\sum_{j=1}^{n} p(j) \log _{2} p(j)
$$

Under the assumption that two time series processes are Markov, Shannon entropy's measure of information flow between these processes is borrowed from the Kullback and Leibler [71] distance model. We present $I$ and $J$ as two discrete random variables with corresponding marginal probabilities of $p(i)$ and $p(j)$, joint probability $p(i, j)$, with dynamic stationary Markov process of order $k$ (process $I$ ) and $I$ (process $J$ ). The Markov property implies that the probability to observe $I$ at time $t+1$ in the state $i$ conditional on the $k$ previous observations is $p\left(i_{t+1} \mid i_{t}, \ldots\right.$, $\left.i_{t-k+1}\right)=p\left(i_{t+1} \mid i_{t}, \ldots, i_{t-k}\right)$. To encode the observation at $t+1$, the average bits number needed before $k$ values is known, which can be illustrated as

$$
h_{j}(k)=-\sum_{i} p\left(i_{t+1}, i_{t}^{(k)}\right) \log _{2} p\left(i_{t+1} \mid i_{t}^{(k)}\right),
$$

where $i_{t}^{(k)}=\left(i_{t}, \ldots, i_{t-k+1}\right)$ (analogously for process $\left.J\right)$. In a bivariate case, relying on the Kullback-Leibler distance, the information flow from process $J$ to process $I$ is measured by quantifying the deviation from the generalised Markov property $p\left(i_{t+1} \mid i_{t}^{(k)}\right)=p\left(i_{t+1} \mid i_{t}^{(k)}, j_{t}^{(I)}\right)$. The Shannon transfer entropy is then given as

$$
T_{J \longrightarrow I}(k, l)=\sum P\left(i_{t+1}, i_{t}^{(k)}, j_{t}^{(I)}\right) \log \frac{P\left(i_{t+1} \mid i_{t}^{(k)}, j_{t}^{(I)}\right)}{P\left(i_{t+1} \mid i_{t}^{(k)}\right)},
$$

where $T_{J \longrightarrow I}$ calculates the information flow from $J$ to $I$. Conversely, the information flow from $I$ to $J, T_{I \longrightarrow J}$, can be derived. The dominant direction of the information flow can be referred to as the net information flow calculated as the difference between $T_{J \longrightarrow I}$ and $T_{I \longrightarrow J}$.

While the Shannon entropy is useful, in the financial setting, it is lacking in assigning equal weights to all possible realisation in the specified probability distribution. This assumption does not account for heavy tails which are rife in asset prices and returns. However, the Rényi [55] transfer entropy (RE) solves this problem through a weighting parameter $q$. The RE can be calculated as

$$
H_{J}^{q}=\frac{1}{1-q} \log _{2} \sum_{j} P^{q}(j)
$$

with $q>0$. For $q \longrightarrow 1$, RE converges to Shannon entropy. For $0<q<1$, thus, low probability events receive more weight, while for $q>1$, the weights benefit outcomes $j$ with a higher initial probability. As a result, Rényi entropy allows for emphasis to be placed on different portions of the distribution, depending on parameter $q[13,68]$. This is the desirable feature of the RE financial applications, as against the Shannon entropy. 
Further, with the escort distribution, $\varnothing_{q}(j)=p^{q}(j) /$ $\sum_{j} p^{q}(j)$ for $q>0$ to normalise the weighted distributions [72], RE is derived as

$$
\mathrm{RE}_{J \longrightarrow I}(k, l)=\frac{1}{1-q} p\left(i_{t+1}, i_{t}^{(k)}, j_{t}^{(I)}\right) \log _{2} \frac{\sum_{i} \varnothing_{q}\left(i_{t}^{(k)}\right) P^{q}\left(i_{t+1} \mid i_{t}^{(k)}\right)}{\sum_{i, j} \varnothing_{q}\left(i_{t}^{(k)}, j_{t}^{(I)}\right) P^{q}\left(i_{t+1} \mid i_{t}^{(k)}, j_{t}^{(I)}\right)}
$$

Note that the calculation of the Rényi transfer entropy can result in negative values. In such a situation, knowing the history of $J$ depicts even greater uncertainty than would otherwise be indicated by only knowing the history of $I$ alone. In the application of our study, negative values depict higher risk while positive values indicate lower risks.

In small samples, transfer entropy estimates tend to be biased [73]. This can be corrected by calculating the effective transfer entropy (ETE) as

$$
\operatorname{ETE}_{J \longrightarrow I}(k, l)=T_{J \longrightarrow I}(k, l)-T_{\text {Jshuffled } \longrightarrow I}(k, l),
$$

where $T_{J \text { shuffled } \longrightarrow I}(k, l)$ denote the transfer entropy using shuffled versions of the time series $J$. By repeated random drawings of the observed time series $J$ and realigning them to generate a new time series, the process destroys the time series serial dependencies of $J$, while keeping any statistical dependencies between $J$ and $I$. This directs $T_{\text {Jshuffled } \longrightarrow I}(k, l)$ to converge to zero with an increasing sample size, and any nonzero value of $T_{\text {Jshuffled } \longrightarrow I}(k, l)$ is due to small sample effects. Thus, repeated shuffling and the average of the resulting shuffled transfer entropy estimates across all replications serve as an estimator for the small sample bias. These are subtracted from the RE estimate to obtain bias-corrected effective transfer entropy estimates.

The statistical significance of the transfer entropy estimates can be determined using the Markov block bootstrap technique. This preserves the dependencies within the variables $J$ and $I$ but eliminates the statistical dependencies between them unlike shuffling. Thus, bootstrapping provides a distribution of transfer entropy estimates to be tested under the null hypothesis of no information flow. The associated $p-$ value is given by $1-$ $\widehat{q} \mathrm{~T}$, where $\hat{q} \mathrm{~T}$ denotes the quantile of the simulated distribution that is determined by the respective transfer entropy estimate [68].

Last, since transfer entropy algorithms are originally based on discrete data, continuous data used in the framework need to be discretised. This can be done by partitioning the data into a finite set of bins; the process is known as symbolic encoding [68]. For a number of bins $n$, with bounds $q_{1}, q_{2}, q_{3}, \ldots, q_{n-1} \quad\left(q_{1}<q_{2}<\right.$ $\left.q_{3}<\cdots<q_{n-1}\right)$ and continuous observed time series data $y_{t}$, the symbolically encoded time series (i.e., discrete) can be given as

$$
S_{t}= \begin{cases}1, & y_{t} \leq q_{1}, \\ 2, & q_{1}<y_{t}<q_{2}, \\ \vdots & \\ n-1, & q_{n-2}<y_{t}<q_{n-1}, \\ n, & y_{t} \geq q_{n-1} .\end{cases}
$$

The choice of the number of bins must be informed by the size and distribution of the observed time series. Since tail observations are of importance, binning is usually based on the empirical quantiles of the left and right tails. This is easily achieved by choosing the 5\% and 95\% empirical quantiles as lower and upper bounds of the bins. This results in three symbolic encodings where the first bin (5\%) comprises negative extreme returns (i.e., lower tail), the third bin (95\%) captures positive extreme returns (upper tail), and the second bin (middle 90\%) contains the normal returns. By making use of the chain rule on the symbolic encoding, conditional probabilities can be written as fractions of joint probability. In that case, the probabilities in equations (9) and (11) can be computed by the relative frequencies of all possible realisation. See Behrendt et al. [68] for a complete description.

\section{Data and Preliminary Analysis}

We used daily closing prices of the 27 world equity indices from 20 countries, 4 economic blocs (Developed Markets, Emerging Markets, Europe, Middle East, Africa (EMEA), and Eurozone), and the Nasdaq Global Equity Index. We track the information transfer from the daily global confirmed cases of COVID-19 from December 31, 2019, to April 18,2021 . The data on equity indices and COVID-19 (see Table 2) were retrieved from the EquityRT and Our World in Data (https://ourworldindata.org/coronavirus) platforms, respectively. Some of the selected indices are overlapping in the sense that some are subsets of others. This is helpful to ascertain both aggregate and disaggregated impact of COVID-19. It is obvious that all the indices are subsets of the Global Index. The descriptive statistics of the returns of all equity indices and confirmed COVID-19 cases are presented in Table 2. The returns were calculated as the difference of logarithms for consecutive closing indices as $r_{t}=\log \left(P_{t+1}\right)-\log \left(P_{t}\right)$, where $r_{t}$ is the return from period $t$ to $t+1$ and $P_{t}$ and $P_{t+1}$ are observations at periods $t$ and $t+1$, respectively. To facilitate comparison, all the series have been matched by date to balance. 
Table 2: Descriptive statistics of equity return and COVID-19 confirmed cases.

\begin{tabular}{|c|c|c|c|c|c|c|}
\hline Country (equity index) & Min. & Max. & Mean. & Std. dev. & Skewness & Kurtosis these are excess kurtosis values \\
\hline Argentina (Merval) & -0.1587 & 0.0962 & -0.0030 & 0.0303 & -0.5499 & 4.0402 \\
\hline Australia (ASX 200) & -0.1122 & 0.0755 & 0.0003 & 0.0193 & -1.3039 & 9.3884 \\
\hline Belgium (BL20) & -0.1618 & 0.0629 & 0.0010 & 0.0194 & -2.6899 & 23.4941 \\
\hline Brazil (Ibovespa) & -0.2038 & 0.1608 & -0.0009 & 0.0322 & -1.0793 & 10.7386 \\
\hline Canada (TSX Comp. Index) & -0.1372 & 0.0863 & 0.0011 & 0.0189 & -2.3079 & 18.9526 \\
\hline China (SSE Comp. Index) & -0.0472 & 0.0621 & 0.0007 & 0.0116 & 0.5450 & 4.4084 \\
\hline Eurozone (EuroStoxx50) & -0.1409 & 0.0628 & 0.0011 & 0.0182 & -2.3791 & 18.0107 \\
\hline France (CAC 40) & -0.1395 & 0.0740 & 0.0011 & 0.0185 & -2.0936 & 16.9682 \\
\hline Germany (DAX) & -0.1391 & 0.0493 & 0.0011 & 0.0181 & -2.4053 & 17.3262 \\
\hline Hong Kong (Heng Seng Index) & -0.0574 & 0.0377 & -0.0002 & 0.0132 & -0.5067 & 2.2285 \\
\hline India (BSE SENSEX) & -0.0932 & 0.0947 & 0.0018 & 0.0190 & -0.2215 & 6.2844 \\
\hline Japan (Nasdaq Index) & -0.0668 & 0.0684 & 0.0005 & 0.0137 & -0.1635 & 6.1673 \\
\hline Mexico (Nasdaq Index) & -0.1532 & 0.1106 & -0.0001 & 0.0322 & -0.8090 & 4.3852 \\
\hline New Zealand (Nasdaq Index) & -0.0866 & 0.0652 & 0.0016 & 0.0162 & -0.6615 & 5.9766 \\
\hline Russia (Moex Russia Index) & -0.0909 & 0.0504 & 0.0005 & 0.0197 & -0.5886 & 2.2965 \\
\hline South Africa (FTSE/JSE Index) & -0.1264 & 0.0721 & 0.0016 & 0.0215 & -1.0916 & 7.1083 \\
\hline South Korea (KOSPI) & -0.1147 & 0.0861 & 0.0009 & 0.0198 & -1.1480 & 7.1550 \\
\hline Taiwan (TAIEX Index) & -0.0676 & 0.0376 & 0.0008 & 0.0124 & -1.1693 & 4.9676 \\
\hline UK 1 (CBOE 100$)$ & -0.1290 & 0.0558 & 0.0003 & 0.0180 & -1.7788 & 12.8458 \\
\hline US 1 (S\&P 500) & -0.0999 & 0.0888 & 0.0011 & 0.0179 & -0.4174 & 8.2283 \\
\hline UK 2 (Nasdaq) & -0.1404 & 0.0551 & 0.0005 & 0.0180 & -2.3834 & 18.0158 \\
\hline US 2 (Nasdaq) & -0.0991 & 0.0893 & 0.0012 & 0.0196 & -0.4722 & 5.2161 \\
\hline Nasdaq Dev. Mkts & -0.1034 & 0.0514 & 0.0011 & 0.0149 & -1.8690 & 13.3438 \\
\hline Nasdaq EM & -0.0790 & 0.0472 & 0.0008 & 0.0136 & -1.1082 & 6.7668 \\
\hline Nasdaq EMEA & -0.1385 & 0.0431 & 0.0010 & 0.0166 & -3.2909 & 24.8351 \\
\hline Nasdaq Eurozone & -0.1507 & 0.0486 & 0.0012 & 0.0179 & -3.2665 & 25.4667 \\
\hline Nasdaq Global & -0.1219 & 0.0777 & 0.0015 & 0.0249 & -0.9766 & 4.0239 \\
\hline COVID-19 & 0.0026 & 0.2890 & 0.0215 & 0.0330 & 4.3780 & 24.7706 \\
\hline
\end{tabular}

Before we delve into the statistical distribution of returns, it is appropriate to have a quick look at the behavioural trajectories of the indices under consideration and COVID-19 confirmed cases. Figure 2 shows the graphical representation of the trend of the time series of the indices of the 27 stock markets and COVID-19 confirmed cases over the study period. A glance shows that all the indices saw a sharp downward trend in the early days of the pandemic which was rising quickly. While the COVID-19 confirmed cases have kept rising until now, more calm returned to the markets as we see upward trends as many countries embarked on lockdown from early February of 2020. However, the markets started to dip again when most countries came out of lockdown, and international travels resumed gradually beginning October 2020. This resulted in a second wave of the virus leading up to the Chistmas festivities. [74] Conversely, there has been an upward trend in the markets after the Christmas season as fears of the second wave abated. The downward trend of the indices reflects the investors and economic reaction to catastrophic events such as pandemics [32]. It is natural to see the inverse relationship between pandemic and financial markets.

A cursory glance at the descriptive statistics of the returns presented in Table 2 reveals interesting features of the return rates of the included stock markets. All 27 indices had positive means in the period considered, except for Argentina, Brazil, Hong Kong, and Mexico. Thus, on average only a few countries have had losses on their equity markets due to COVID-19. In terms of skewness, all countries record higher losses more frequently than higher gains, which is indicated by negative skewness, except for China. This not surprising because China is one of a few countries which have managed the pandemic effectively despite being its origin.

Currently, China ranks 98 out of 221 countries in confirmed cases (https://www.worldometers.info/ coronavirus/countries-where-coronavirus-has-spread/).

Many countries have recorded only a few hundred cases after the 196th rank and many bottom countries have singledigit confirmed cases. These explain why China's equity market could record higher returns than lower returns. The returns of all assets showed excess kurtosis. This implies that the returns are heavy-tailed relative to the normal distribution. This is not surprising, as it is a well-known stylised fact about financial assets [75].

\section{Analysis of Rényi Entropy Results}

At this stage, we address the main objective of this study. We analyse the information transfer from COVID-19 to the selected global equities. We only examine unidirectional transmission from COVID-19 to the equity markets since the equities do not have the potential to influence COVID19 confirmed cases. Effective transfer entropies (ETEs) from the Rényian entropy framework result in both negative (high risk) and positive (low risk) values. This allows for diversification potential by pairing negative receiving ETEs equities with positive recipients. In line with the stylised facts 

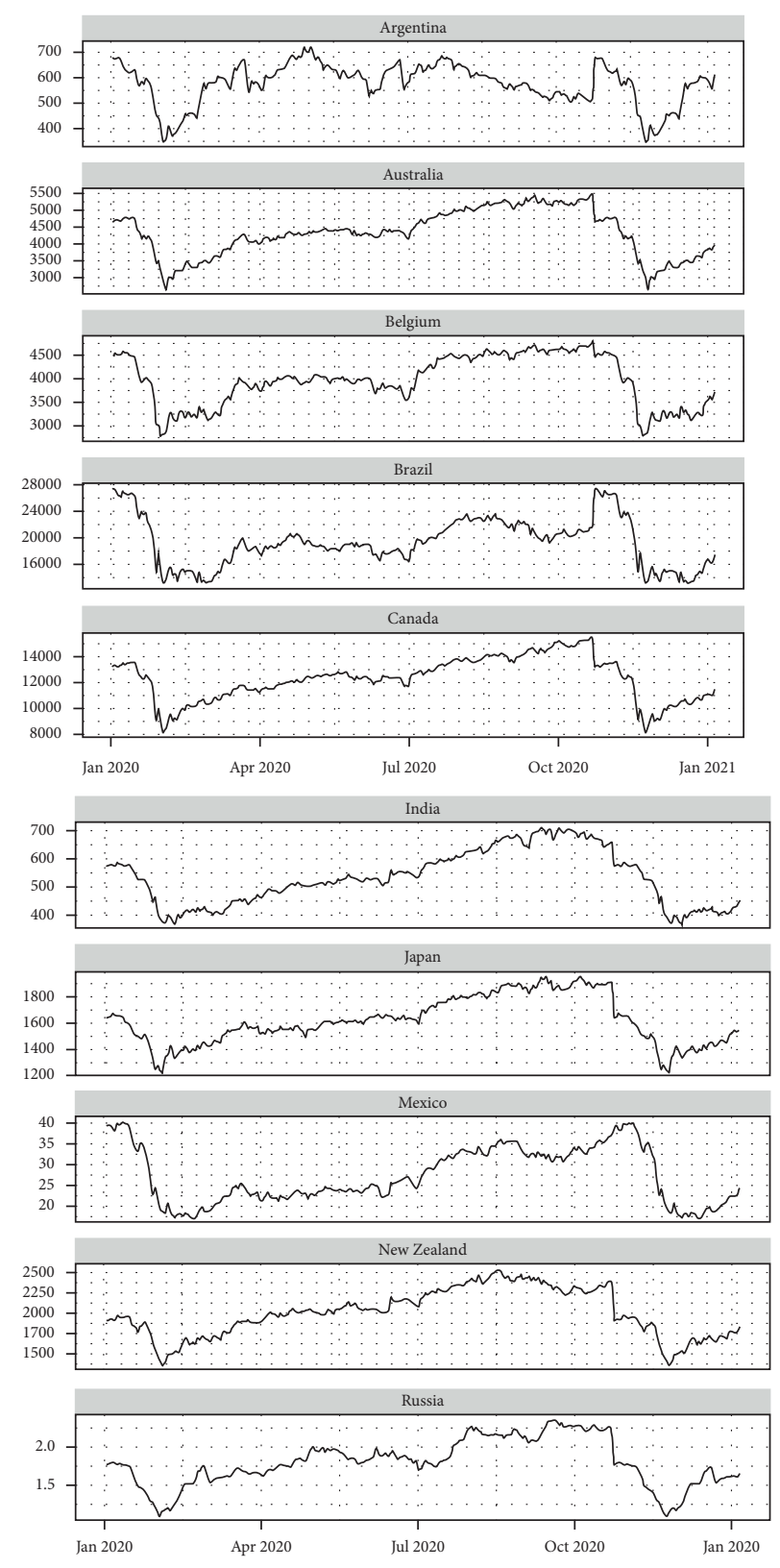
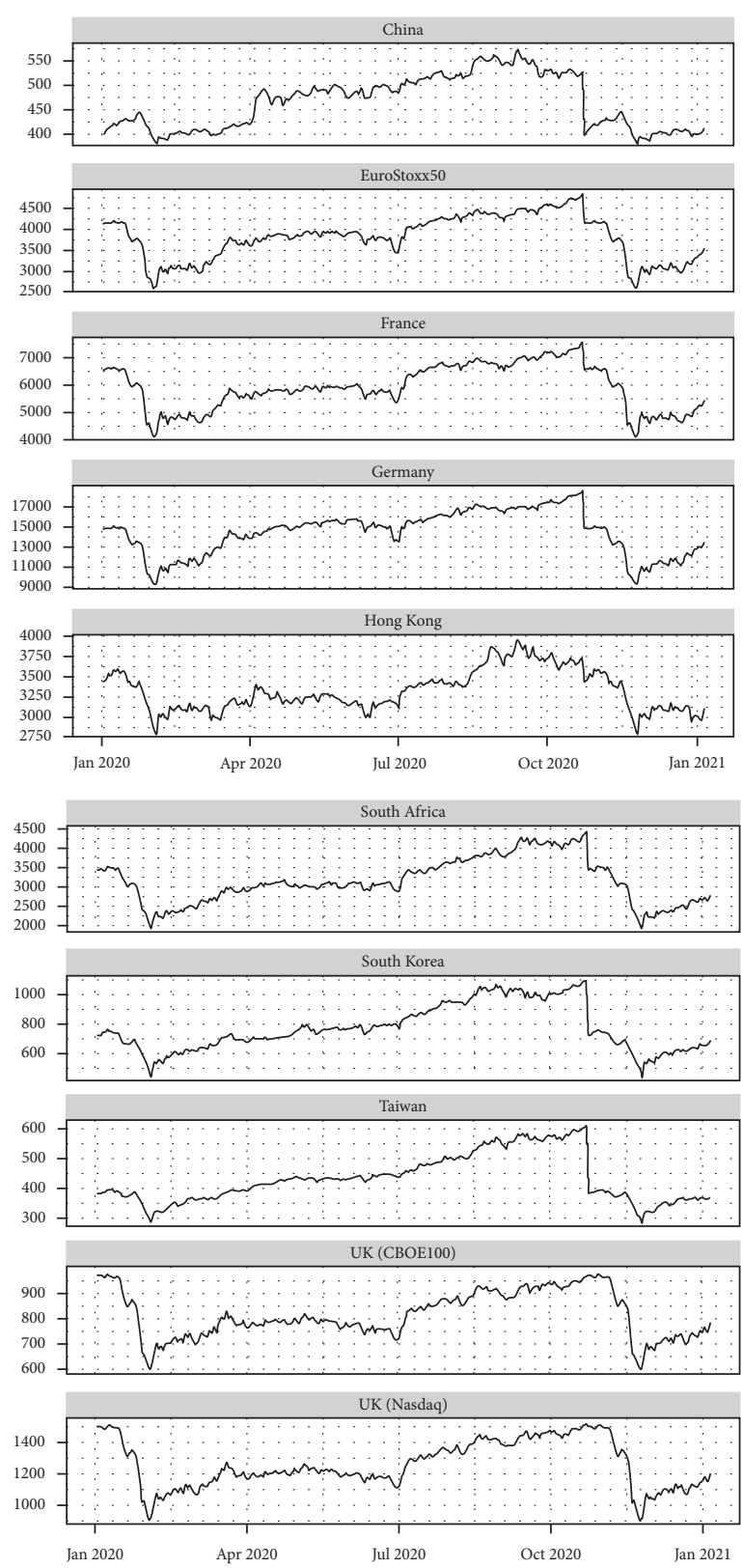

(a)

FIgURE 2: Continued. 

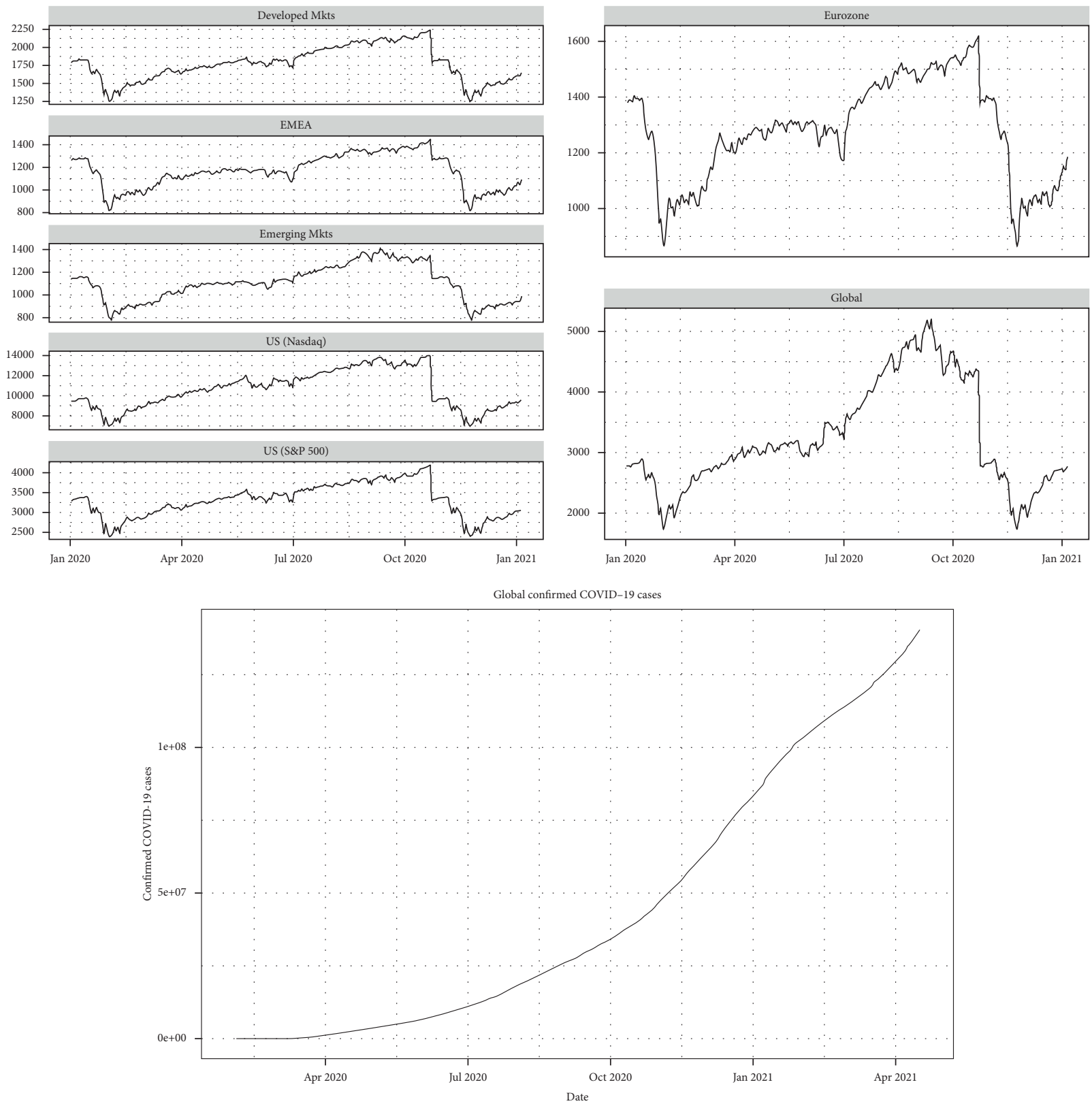

(b)

FIgure 2: Time series plot of COVID-19 and global equities.

of financial returns, we choose the fault weight of 0.30 to account for heavy tails in the equity returns. We also present results for both the composite level and frequency domain. In the latter, intrinsic times are indicated by IMF1 through to IMF6 and Residual. These are representative of short-, medium-, and long-term dynamics, where the residuals denote the long-term trend, which implies the fundamental behaviour of the respective series. These time horizons enable us to analyse the evolving response of the markets to the pandemic.

In Figures 2 (composite level) and 3 (frequency domain), ETEs are indicated by black points inside blue bars. The ends of the blue bars indicate $95 \%$ confidence bounds. For this reason, these confidence bounds should be in either the positive or negative regions for us to fail to accept the null hypothesis of no information flow. Any overlap at the origin shows an insignificant information flow. From Figure 3, we find mostly positive information transfer (ETE) from COVID-19 to the equities, except for Argentina, Brazil, Germany, and Global Index which are negative. Therefore, COVID-19 creates a high risk for Argentina, Brazil, Germany, and the Global Index, but a low risk for the rest.

However, all these ETEs are not statistically significant. Only the Global Index receives a statistically significant negative information flow from COVID-19. However, there are significant positive information flows only to Australia, 


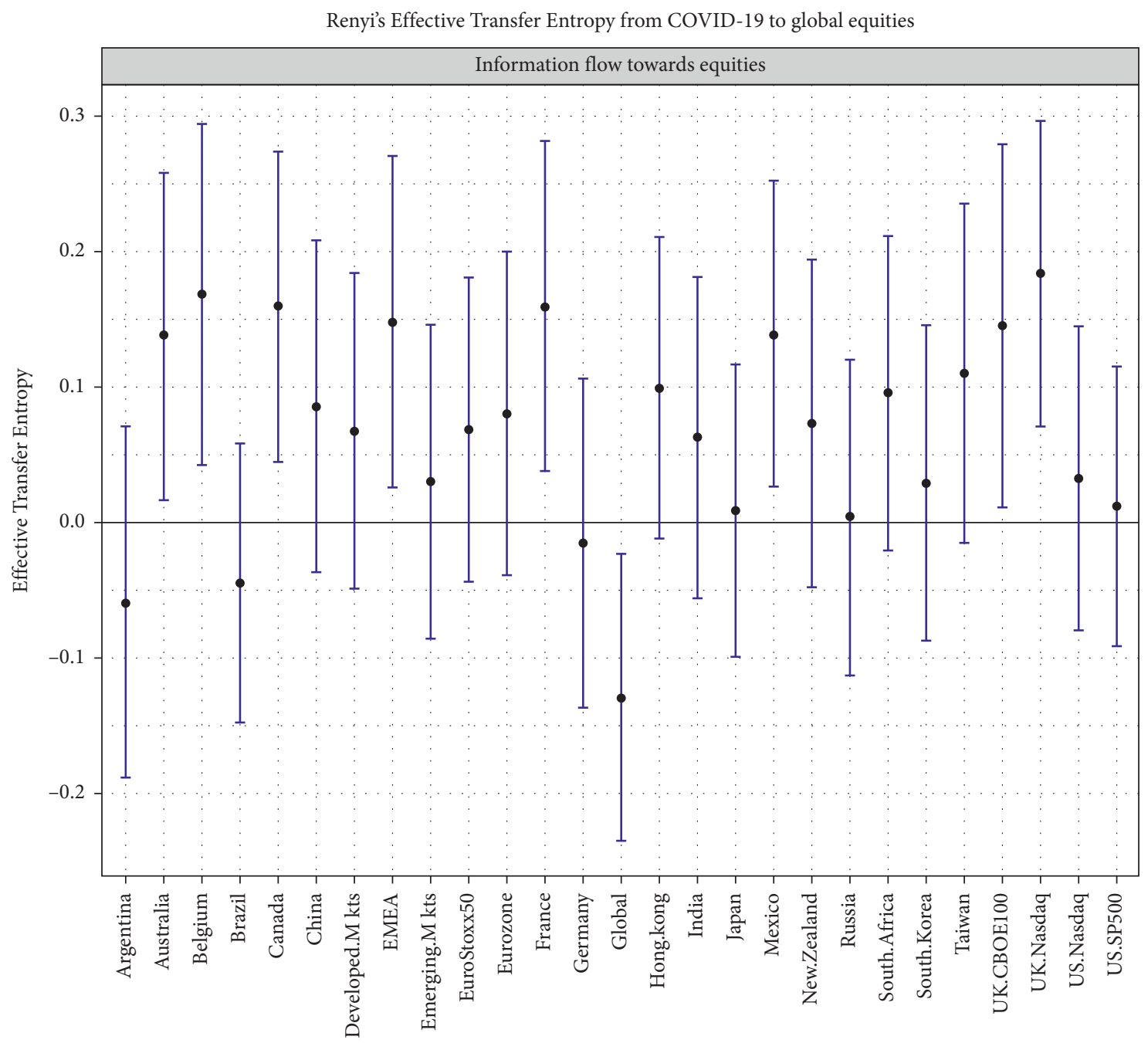

FIgURE 3: Rényian ETE from COVID-19 to global equity markets at the composite level.

Belgium, Canada, EMEA, France, Mexico, UK (CBOE100), and UK (Nasdaq) (recording the highest). The pandemic that fosters high risks in the global equity market is not a surprise. This is a pandemic that has never been seen in modern history and panic associated with it somehow can surpass the real havoc it is inflicting. However, for countries that have managed this well and have stronger financial systems, the composite effect of the pandemic should not be so devastating. This is true as investors tend to take refuge by investing in more advanced and stable economies during crises [76]. We also assume that the impact of COVID-19 is not all doom and gloom, but an avenue to diversify portfolios to reduce risk. Given the negative and positive ETEs, international investors are presented with the opportunity to combine the Global Index as a high-risk asset with any of the equities of Australia, Belgium, Canada, EMEA, France, Mexico, UK (CBOE100), and UK (Nasdaq) to mitigate their risk exposure.

From Figure 4, in the short term of IMF1, there is an increase in the number of equities that receive negative information flow (7: Brazil, Canada, Germany, Global, India, Russia, South Korea, and Taiwan) compared to the composite series (4: Argentina, Brazil, Germany, and Global). Among these, on the ETE to Global Index, Argentina, China (highest), UK (CBOE100), and UK (Nasdaq) are significant. Hence, the Global Index could be bundled with the positive recipients to mitigate risks. At IMF2, negative receiving equities increased to 18 out of 27, but only 3 (Global, Hong Kong (highest), and Mexico) are significant. Given that there are no significant lower-risk assets, no diversification avenue exists at this scale. The pattern holds for IMF3 and IMF4 as well. Negative ETE recipients increase, some significant, while all positive flows are insignificant. These do not offer diversification prospects, as COVID-19 information transmission produces only highrisk assets.

There is a change in the pattern at the medium term of IMF5 where significant positive transmissions are recorded by Canada and New Zealand as negative receipts again by Argentina, Belgium, EuroStoxx50, Germany, India, and Taiwan. It is clear that combinations can help reduce portfolio risk. However, at the long-term scales of IMF6 and Residual diversification potential vanish [77]. In IMF6, all markets receive negative information flows from the 

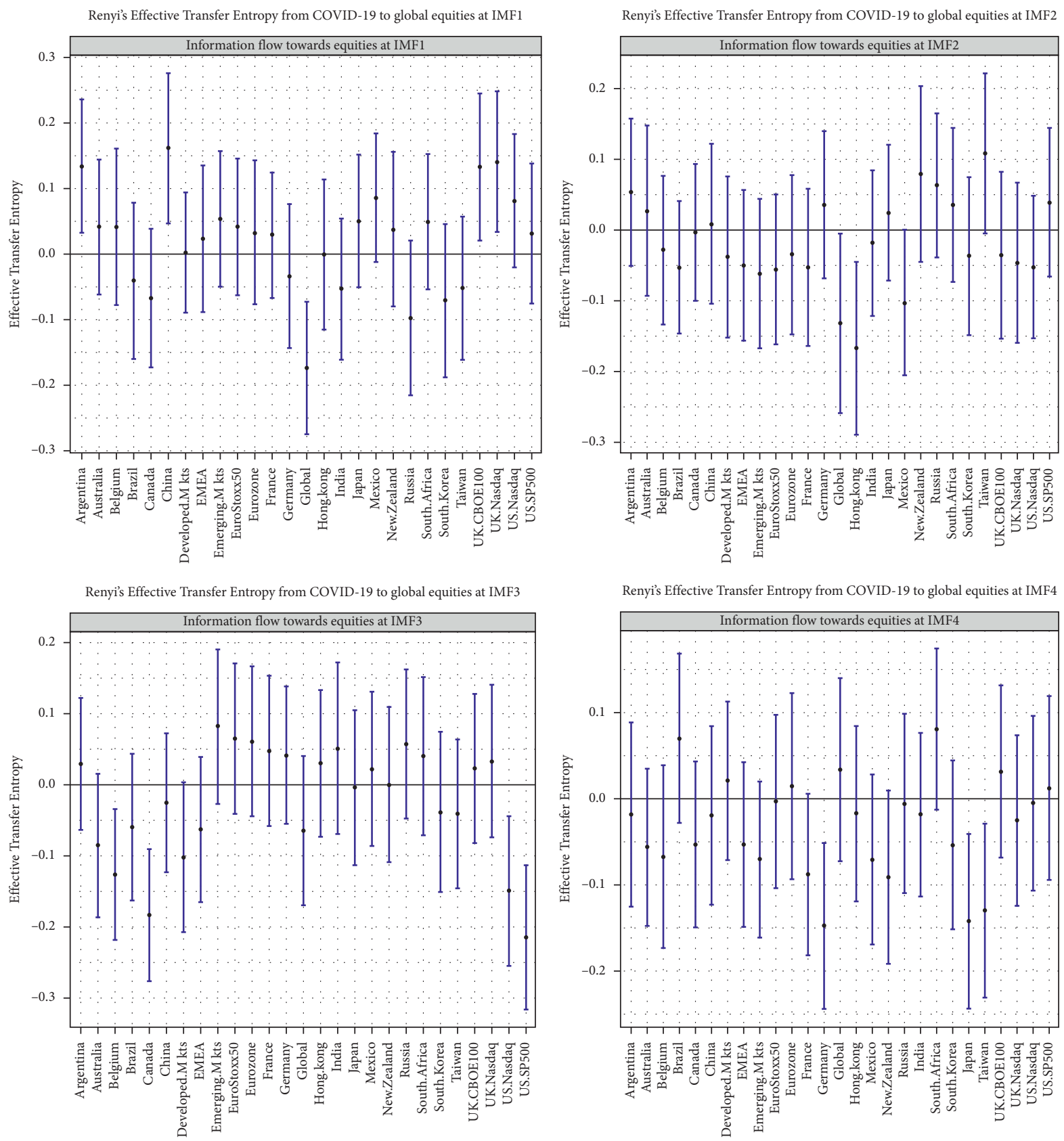

(a)

FIgURE 4: Continued. 

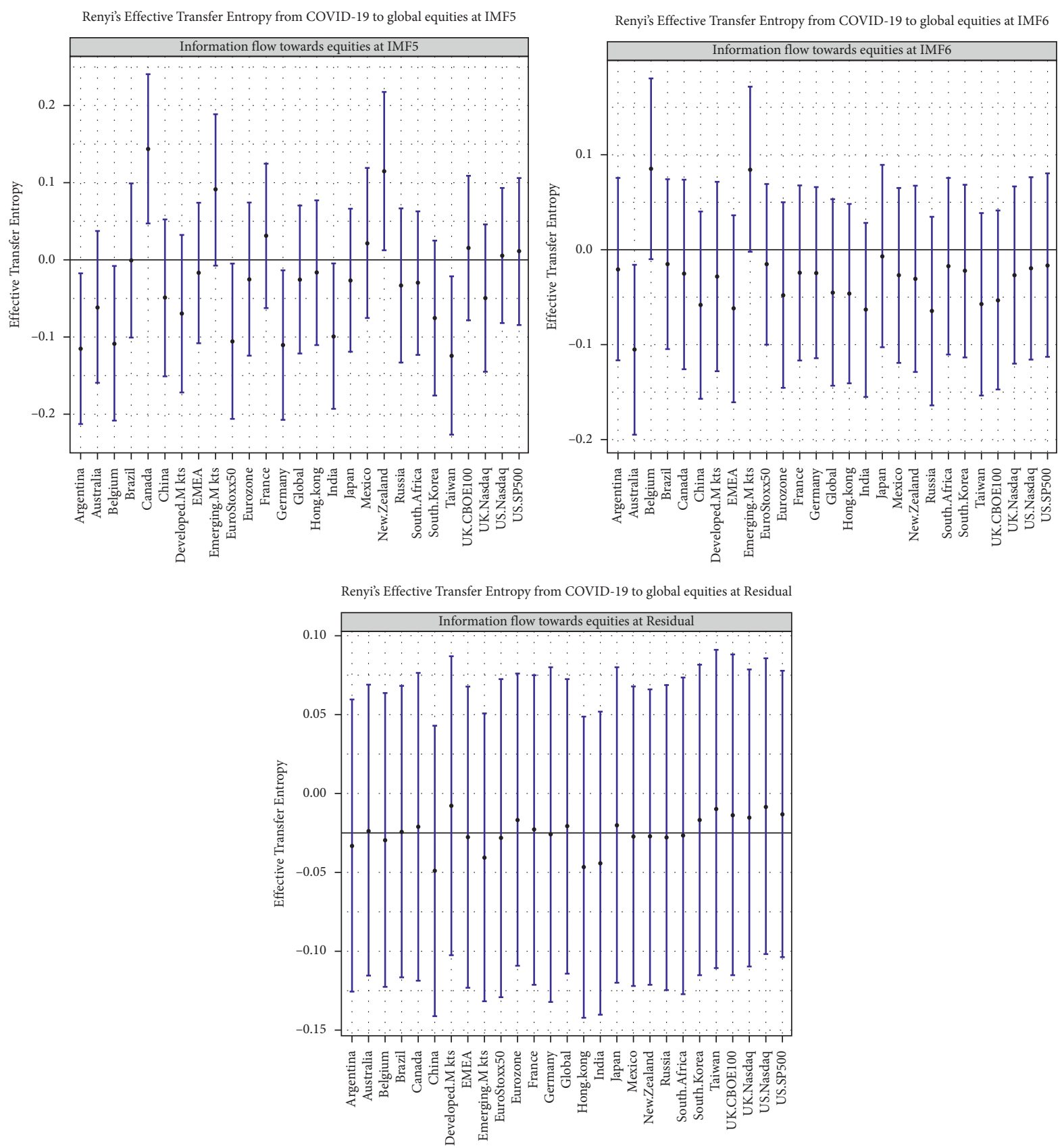

(b)

FIgURE 4: Rényian ETE from COVID-19 to global equity markets in frequency domain.

COVID-19 pandemic, except for Belgium and emerging markets, but only Australia's is statistically significant. Contrary to EMH, the differing transfer entropies of positive and negative across different time scales confirm the adaptive market hypothesis (AMH) of Lo [9], heterogeneous market hypothesis (HMH) of Müller et al. [10], and alternative hypothesis to market efficiency by Cornell [11]. In addition, the results confirm the competitive market hypothesis $(\mathrm{CMH})$ proposed in this study. In all, the results suggest that investors adapt to market dynamics-based sentiment, risk, and reward preferences across time and assets in search for competition compensations to satisfy their goals.

When the fundamental structure of the equity markets is revealed at the Residual scale, all COVID-19 transmits only negative information to all the COVID-19 pandemic but none of them is statistically significant. While the negative flows do not come as surprise, their insignificance communicates an important message to all stakeholders in the financial market. Fundamentally, the pandemic (the number of global infections and the fear associated with it) does not affect the equity markets. We can consider short-term and 
medium-term transmissions as the products of irrational investor behaviour which many see as noise [11, 52]. We purport that in the long term investors may have allayed their fears and rebalanced their portfolios only marginally incorporating the news about the pandemic. For prospective investors and policymakers, the pandemic may not be used as an important variable in arriving at investment and policy decisions concerning global equities. The reason is that the pandemic does not communicate any risks attributes (neither positive nor negative) of the equities to be used in investment and allocation of resources across assets as well as COVID-19 relief packages to the financial or equity markets. In many aspects, this paradigm reflects the efficient market hypothesis (EMH) by Fama $[5,6]$ in the sense that the rationality of the market participants is revealed in spite of the ensuing pandemic. This also confirms the long-term market efficiency [6].

In the spirit of the existing literature, we consider IMF1, IMF2, and IMF3 to represent the short term, IMF4 and IMF5 to capture the medium term, and IMF6 and Residual to denote long-term dynamics, respectively [25, 58, 78, 79]. Yang et al. [58] interpret the short term as a period driven by investor sentiments and market microstructure, the medium term as representing the effect of significant events, and the long term as representative of fundamental values. These demarcations imply that our results are mixed in terms of diversification potentials, being present and absent simultaneously in the short and medium term, as well as at the composite scale. The summary of the results is presented in Table 1 . We also note that, although they vary in magnitude, equity markets receiving both positive and negative significant ETEs are from a mixture of countries and regions that are hard vis-à-vis soft hit by COVID-19 with respect to confirmed cases and fatalities https://www.worldometers. info/coronavirus/countries-where-coronavirus-has-spread/. Hence, it may suggest that while we are in the midst of a global pandemic, COVID-19 is not the only force driving the equity markets. Countries and economic blocs already have underlying fundamentals which determine their performance to a large extent. It is also natural to assume that the extent of the impact of COVID-19 on an equity market is a function of the economic, financial, social, health, technological, and environmental fabric of its host economy. We find empirical support for this analogy in [80] that economies with strong institutions and macroeconomic fundamentals are less impacted by the pandemic.

In the empirical literature, our frequency domain findings corroborate and contradict several studies. The asymmetry in the flow of information in the equity markets is consistent with the sentiment and the connectivity of return and volatility among global equities $[49,50]$. The insignificant ETEs in the long term contradict the findings of [81] that connectedness across financial markets is largely driven by the COVID-19 pandemic. However, the relatively limited diversification prospects and pronounced insignificant entropies for this study confirm the evidence from Lahmiri and Bekiros [49] that international equity markets have not changed in the level of stability but have only become more irregular. Further, pertaining to alternating significant and insignificant transfer entropies between the composite series and the IMFs, we corroborate the weak fleeing impact of the COVID-19 pandemic on global equities as shown in Ahundjanov et al. [82]. They find that a unit increase in COVID-19 popularity of COVID-19 (as measured by related Google search queries) is accompanied by a cumulative decline of 0.038 to $0.069 \%$ in global financial indices after one day and a decline of 0.054 to $0.150 \%$ after one week, after controlling for confirmed cases of COVID19. Nonetheless, Lahmiri and Bekiros [47] find a stronger effect of COVID-19 on equity markets than on energy markets using the GARCH-based Shannon entropy approach. The disparity can be ascribed to the methodological paradigms used.

4.1. Analysis of Shannon Entropy Results. The results of the Shannon ETE in totality confirm the desirability of the Rényi ETE. It is important to note that since the Shannon entropy assigns equal weights to the tails of the return distribution. Hence, we are not surprised that the results do not corroborate the stylised facts of asset returns. The outputs in Figure 1 indicate that there are no significant negative transfer entropies from COVID-19 to the global equities. Where they are significant, only positive information flows are recorded. These imply only high risks in assets regardless of investment horizon. They are also suggesting no diversification potential within the set of global assets.

Furthermore, we find that, at the composite level, the highest number of equity markets receive significant ETEs. The number of significant ETEs tends to reduce with increasing frequency. In the long term, there is not significant ETE to any market at all. In general, these results contradict the AME, HMH, and CMH. However, they align with the $\mathrm{EMH}$ in that all the markets are responding in the same way to the COVID-19 pandemic across all investment horizons. Furthermore, we find that, in the long term, there is a convergence between the Rényi and Shannon entropies, and both confirm the EMH. These indicate that the markets cease to react to the COVID-19 pandemic and revert their fundamental dynamics. The summary of the Shannon entropy results is also presented in Table 1.

\section{Conclusions and Recommendations}

This study examined the possible connection between the COVID-19 infection rate and global equity markets to allow further inferences on how the COVID-19 pandemic could affect global stock markets. First, we decomposed the returns of 27 global stock market indices using the CEEMDAN technique into intrinsic time of short-, medium-, and longterm scales. This helps to understand the dynamic behaviour of the investors' response to the pandemic while removing the noise from the series. This approach appeals to the AMH, $\mathrm{HMH}$, and AHME, all in opposition to the EMH. The EMH has also been confirmed to decline sharply and persists for the S\&P 500 index in the early stages of the pandemic, as shown in Wang and Wang [48]. Second, we employ the Rényi transfer entropy on both composite series and their 
frequency domain counterparts to quantify the flow of information from COVID-19 to the equity markets. This method accounts for heavy tails in the asset returns while distinguishing between high-risk (negative ETEs) and lowrisk (positive ETEs) assets because of the COVID-19 pandemic. For the purposes of comparison and confirmation we also employed the Shannon transfer entropy to the data. Our data span December 31, 2019, through April 18, 2021.

With Rényi transfer entropy, our results confirm EMH in the long term, but in the short and medium term, they confirm the AMH, HMH, and AHME which disagree with the EMH. The summary of our empirical results is that, in the long run, both the Rényi and Shannon transfer entropies fail to reject the null hypothesis of no information flow from COVID-19 to the selected global equity markets. At the time horizon, the fundamental dynamics of the markets are at play. Thus, there exists no potential for diversification across the equity markets. This corroborates the existing empirical literature but for a different reason. The common reason for no diversification avenue in the long term is a high level of integration among markets; however, in this, it is for the fact that the market is saturated with the needed information to make rational decisions. Therefore, the information transmitted by the COVID-19 pandemic does not influence investors' choices. Nevertheless, in terms of the Shannon entropy, there are no diversification potentials because all significant entropies are positive across the board.

However, the Rényian entropies show a different outlook. In the short and medium terms, there is a mixed result of significant and insignificant information flows (both positive and negative) for several equities. Except in the long term, all information flows to the Global Index were significant and negative. This indicates an overall high-risk perception of the global equity market due to the COVID-19 pandemic. On the bright side, this pattern offers diversification potential to low-risk equities during the period, notable among them are those from Canada, New Zealand, China, Argentina, and the UK, EMEA, France, Belgium, and Mexico, at different scales. We note that only significant negative flows were recorded for the US. The response of the US to the pandemic, especially at the early stages, was not optimal mainly due to political differences [83]. They may still be hanging over them, as seen in the negative flows.

In the composite series, we find a wider array of low-risk equities (9) to diversify across the Global Index compared to only 4 at IMF1 and 6 at IMF5 (refer to Table 1). It is clear that diversification prospects are generally limited as they occur at only 2-time scales (IMF1 and IMF5) out of a total of 6time scales. These confirm, to some extent, that investor expectations have not been strongly influenced by the pandemic.

Our study has made a significant contribution to the fledgling empirical literature on the impact of COVID-19 in the finance ecosystem. We provide both corroborative and contradictive evidence. In the former, the results conform to stronger and slim (to none) diversification prospects in the short term and long term, respectively. However, in the latter, the reasons are not due to weak versus strong comovements or spillovers but due to quantifiable, significant, or insignificant asymmetric (positive or negative) information flows from COVID-19 to the respective equity markets. Specifically, our CEEMDAN-based transfer entropy methodology sheds new light on diversification potential in the frequency domain. In a noise-free environment, the direction of diversification is further informed by low-risk vis-à-vis high risk as suggested by positive and negative effective transfer entropies, respectively. In the end, investors are equipped with the knowledge to make decisions by taking cognisance of time horizon, the type of information flow, and the reason for the existence of diversification prospects. Similarly, policymakers can formulate and implement actions with respect to these tenets. This suggestion reiterates [84] that both investors and policymakers need to be careful against sentimental shocks and decisions. The findings have implications for the world stock markets in the sense that the propensity of COVID-19 to escalate and cause irreversible damage to global financial markets is relatively small. In contrast to Akhtaruzzaman et al. [38] and Yu et al. [85], our results do not make any attributions to financial contagion emanating from the COVID-19 pandemic. However, a long lifespan of the pandemic may have lasting effects on the global financial markets [86].

\section{Data Availability}

The data used in this paper are publicly available. Since this study used secondary data publicly available, the authors, therefore, decided not to reshare.

\section{Conflicts of Interest}

The authors declare no conflicts of interest.

\section{References}

[1] M. Roser, H. Ritchie, E. Ortiz-Ospina, and J. Hasell, "Coronavirus Pandemic (COVID-19): our world data," 2020, https://ourworldindata.org/coronavirus.

[2] N. J. Gormsen and R. Koijen, "Coronavirus: impact on stock prices and growth expectations," 2020, https://voxeu.org/ article/coronavirus-impact-stock-prices-and-growthexpectations.

[3] S. Ramelli and A. F. Wagner, Feverish Stock Price Reactions to COVID-19, https://ideas.repec.org/p/chf/rpseri/rp2012.html, Swiss Finance Institute, Zürich, Switzerland, 2020, https:// ideas.repec.org/p/chf/rpseri/rp2012.html.

[4] H. Herron and V. Hajric, “The Market's in Panic Mode.' Stock Markets Plunge 12\% Amid Coronavirus Fears (Time)," 2020, https://time.com/5803847/coronavirus-stocks-fall/.

[5] E. F. Fama, "Efficient capital markets: a review of theory and empirical work," The Journal of Finance, vol. 25, no. 2, pp. 383-417, 1970.

[6] E. F. Fama, "Market efficiency, long-term returns, and behavioral finance," Journal of Financial Economics, vol. 49, no. 3, pp. 283-306, 1998.

[7] M. Jensen, "Some anomalous evidence regarding market efficiency," Journal of Financial Economics, vol. 6, no. 2-3, pp. 95-101, 1978. 
[8] F. S. Mishkin and S. Eakins, Financial Markets and Institutions, Pearson, New York, NY, USA, 9th edition, 2017.

[9] A. W. Lo, "The adaptive markets hypothesis," Journal of Portfolio Management, vol. 30, no. 5, pp. 15-29, 2004.

[10] U. A. Müller, M. M. Dacorogna, R. D. Davé, O. V. Pictet, R. B. Olsen, and J. R. Ward, "Fractals and intrinsic time: a challenge to econometricians," Unpublished Manuscript, Olsen Associates, Zürich, Switzerland, 1993.

[11] B. Cornell, "What is the alternative hypothesis to market efficiency?" Journal of Portfolio Management, vol. 44, no. 7, pp. 3-6, 2018.

[12] D. G. Baur and B. M. Lucey, "Is gold a hedge or a safe haven? An analysis of stocks, bonds and gold," Financial Review, vol. 45 , no. 2, pp. 217-229, 2010.

[13] A. M. Adam, "Susceptibility of stock market returns to international economic policy: evidence from effective transfer entropy of Africa with the implication for open innovation," Journal of Open Innovation: Technology, Market, and Complexity, vol. 6, no. 3, p. 71, 2020.

[14] A. M. Adam, K. Kyei, S. Moyo, R. Gill, and E. N. Gyamfi, "Similarities in southern african development community (SADC) exchange rate markets structure: evidence from the ensemble empirical mode decomposition," Journal of African Business, pp. 1-16, 2021.

[15] D. G. Baur and T. K. McDermott, "Is gold a safe haven? International evidence," Journal of Banking \& Finance, vol. 34, no. 8, pp. 1886-1898, 2010.

[16] D. G. Baur and T. K. J. McDermott, "Why is gold a safe haven?" Journal of Behavioral and Experimental Finance, vol. 10, pp. 63-71, 2016.

[17] S. Bekiros, S. Boubaker, D. K. Nguyen, and G. S. Uddin, "Black swan events and safe havens: the role of gold in globally integrated emerging markets," Journal of International Money and Finance, vol. 73, pp. 317-334, 2017.

[18] A. Dutta, D. Das, R. K. Jana, and X. V. Vo, "COVID-19 and oil market crash: revisiting the safe haven property of gold and Bitcoin,” Resources Policy, vol. 69, Article ID 101816, 2020.

[19] M. M. Habib and L. Stracca, "Getting beyond carry trade: what makes a safe haven currency?" Journal of International Economics, vol. 87, no. 1, pp. 50-64, 2012.

[20] Y. Huang, K. Duan, and T. Mishra, "Is Bitcoin really more than a diversifier? A pre- and post-COVID-19 analysis," Finance Research Letters, Article ID 102016, 2021.

[21] Y. Jiang, J. Lie, J. Wang, and J. Mu, "Revisiting the roles of cryptocurrencies in stock markets: a quantile coherency perspective," Economic Modelling, vol. 95, pp. 21-34, 2021.

[22] A. Kliber, P. Marszałek, I. Musiałkowska, and K. Świerczyńska, "Bitcoin: safe haven, hedge or diversifier? Perception of Bitcoin in the context of a country's economic situation-a stochastic volatility approach," Physica A: Statistical Mechanics and Its Applications, vol. 524, pp. 246-257, 2019.

[23] W. Mensi, R. Nekhili, X. V. Vo, and S. H. Kang, "Oil and precious metals: volatility transmission, hedging, and safe haven analysis from the Asian crisis to the COVID-19 crisis," Economic Analysis and Policy, vol. 71, pp. 73-96, 2021.

[24] P. Owusu Junior, A. M. Adam, and G. Tweneboah, "Connectedness of cryptocurrencies and gold returns: evidence from frequency-dependent quantile regressions," Cogent Economics \& Finance, vol. 8, no. 1, Article ID 1804037, 2020.

[25] P. O. Junior, A. K. Tiwari, H. Padhan, and I. Alagidede, "Analysis of EEMD-based quantile-in-quantile approach on spot- futures prices of energy and precious metals in India," Resources Policy, vol. 68, Article ID 101731, 2020.
[26] P. Owusu Junior and G. Tweneboah, "Are there asymmetric linkages between African stocks and exchange rates?" Research in International Business and Finance, vol. 54, Article ID 101245, 2020.

[27] L. Yarovaya, R. Matkovskyy, and A. Jalan, "The effects of a "black swan" event (COVID-19) on herding behavior in cryptocurrency markets," Journal of International Financial Markets, Institutions and Money, Article ID 101321, 2021.

[28] D. Acemoglu, S. Johnson, and J. Robinson, "Disease and development in historical perspective," Journal of the European Economic Association, vol. 1, no. 2-3, pp. 397-405, 2003.

[29] D. E. Bloom, J. D. Sachs, P. Collier, and C. Udry, "Geography, demography, and economic growth in Africa," Brookings Papers on Economic Activity, vol. 1998, no. 2, pp. 207-295, 1998.

[30] S. Gallup and D. Mellinger, Geography and Economic Development, Harvard University Press, Cambridge, MA, USA, 1999, https://www.hks.harvard.edu/centers/cid/publications/ faculty-working-papers/geography-and-economicdevelopment.

[31] C. Barrett, K. Bisset, J. Leidig, A. Marathe, and M. Marathe, "Economic and social impact of influenza mitigation strategies by demographic class," Epidemics, vol. 3, no. 1, pp. 19-31, 2011.

[32] B. Sander, A. Nizam, L. P. Garrison, M. J. Postma, M. E. Halloran, and I. M. Longini, "Economic evaluation of influenza pandemic mitigation strategies in the United States using a stochastic microsimulation transmission model," Value in Health, vol. 12, no. 2, pp. 226-233, 2009.

[33] N. D. Schwartz, "Coronavirus recession looms, its course 'unrecognizable'-the New York Times," 2020, https://www. nytimes.com/2020/03/21/business/economy/coronavirusrecession.html.

[34] C. M. Reinhart, "This time truly is different by Carmen M. Reinhart (Project Syndicate)," 2020, https://www.projectsyndicate.org/commentary/covid19-crisis-has-no-economicprecedent-by-carmen-reinhart-2020-03.

[35] J. Schoenfeld, "The invisible risk: pandemics and the financial markets," SSRN Electronic Journal, 2020.

[36] J. W. Goodell and S. Goutte, "Diversifying with cryptocurrencies during COVID-19," SSRN Electronic Journal, 2020.

[37] K. P. Prabheesh, "Dynamics of foreign portfolio investment and stock market returns during the COVID-19 pandemic: evidence from India," Asian Economics Letters, vol. 1, no. 2, Article ID 17658, 2020.

[38] M. Akhtaruzzaman, S. Boubaker, and A. Sensoy, "Financial contagion during COVID-19 crisis," Finance Research Letters, vol. 38, Article ID 101604, 2021.

[39] T. Conlon, S. Corbet, and R. J. McGee, “Are cryptocurrencies a safe haven for equity markets? An international perspective from the COVID-19 pandemic," Research in International Business and Finance, vol. 54, Article ID 101248, 2020.

[40] B. Garg and K. P. Prabheesh, "The nexus between the exchange rates and interest rates: evidence from BRIICS economies during the COVID-19 pandemic," Studies in Economics and Finance, vol. 38, no. 2, pp. 469-486, 2021.

[41] W. Hanif, W. Mensi, and X. V. Vo, "Impacts of COVID-19 outbreak on the spillovers between US and Chinese stock sectors," Finance Research Letters, vol. 40, Article ID 101922, 2021.

[42] M. M. Karim, M. A. F. Chowdhury, and M. Masih, "Re-examining oil and BRICS' stock markets: new evidence from wavelet and MGARCH-DCC," Macroeconomics and Finance in Emerging Market Economies, pp. 1-19, 2021. 
[43] K. Malik, S. Sharma, and M. Kaur, "Measuring contagion during COVID-19 through volatility spillovers of BRIC countries using diagonal BEKK approach," Journal of Economics Studies, 2021.

[44] P. K. Narayan, N. Devpura, and H. Wang, "Japanese currency and stock market-What happened during the COVID-19 pandemic?" Economic Analysis and Policy, vol. 68, pp. 191198, 2020.

[45] L. Yarovaya, A. H. Elsayed, and S. Hammoudeh, "Determinants of spillovers between islamic and conventional financial markets: exploring the safe haven assets during the COVID19 pandemic," Finance Research Letters, vol. 79, Article ID 101979, 2021.

[46] T. Schreiber, "Measuring information transfer," Physical Review Letters, vol. 85, no. 2, pp. 461-464, 2000.

[47] S. Lahmiri and S. Bekiros, "Randomness, informational entropy, and volatility interdependencies among the major world markets: the role of the COVID-19 pandemic," Entropy, vol. 22, no. 8, p. 833, 2020.

[48] J. Wang and X. Wang, "COVID-19 and financial market efficiency: evidence from an entropy-based analysis," Finance Research Letters, Article ID 101888, 2021.

[49] S. Lahmiri and S. Bekiros, "The impact of COVID-19 pandemic upon stability and sequential irregularity of equity and cryptocurrency markets," Chaos, Solitons \& Fractals, vol. 138, Article ID 109936, 2020.

[50] S. Lahmiri and S. Bekiros, "Renyi entropy and mutual information measurement of market expectations and investor fear during the COVID-19 pandemic," Chaos, Solitons \& Fractals, vol. 139, Article ID 110084, 2020.

[51] T. Dimpfl and F. J. Peter, "The impact of the financial crisis on transatlantic information flows: an intraday analysis," Journal of International Financial Markets, Institutions and Money, vol. 31, pp. 1-13, 2014.

[52] H. Hassani, A. Dionisio, and M. Ghodsi, "The effect of noise reduction in measuring the linear and nonlinear dependency of financial markets," Nonlinear Analysis: Real World Applications, vol. 11, no. 1, pp. 492-502, 2010.

[53] F. X. Diebold and K. Yilmaz, "Better to give than to receive: predictive directional measurement of volatility spillovers," International Journal of Forecasting, vol. 28, no. 1, pp. 57-66, 2012.

[54] J. Baruník and T. Křehlík, "Measuring the frequency dynamics of financial connectedness and systemic risk," Journal of Financial Econometrics, vol. 16, no. 2, pp. 271-296, 2018.

[55] A. Rényi, "On measures of information and entropy," in Proceedings of the 4th Berkeley Symposium on Mathematics, Statistics and Probability, vol. 1, p. 547, Berkeley, CA, USA, 1961.

[56] S. I. Ivanov, "The influence of ETFs on the price discovery of gold, silver and oil," Journal of Economics and Finance, vol. 37, no. 3, pp. 453-462, 2013.

[57] J. B. Ramsey and C. Lampart, "The decomposition of economic relationships by time scale using wavelets: expenditure and income," Studies in Nonlinear Dynamics and Econometrics, vol. 3, no. 1, pp. 23-42, 1998.

[58] B. Yang, Y. Sun, and S. Wang, "A novel two-stage approach for cryptocurrency analysis," International Review of Financial Analysis, vol. 72, Article ID 101567, 2020.

[59] Z. Huang, "Extensions to the k-means algorithm for clustering large data sets with categorical values," Data Mining and Knowledge Discovery, vol. 2, no. 3, pp. 283-304, 1998.

[60] P. J. J. Luukko, J. Helske, and E. Räsänen, "Introducing libeemd: a program package for performing the ensemble empirical mode decomposition," Computational Statistics, vol. 31, no. 2, pp. 545-557, 2016.

[61] M. E. Torres, M. A. Colominas, G. Schlotthauer, and P. Flandrin, "A complete ensemble empirical mode decomposition with adaptive noise," in Proceedings of the 2011 IEEE International Conference on Acoustics, Speech and Signal Processing (ICASSP), pp. 4144-4147, Prague, Czech Republic, May 2011.

[62] Q. Peng, F. Wen, and X. Gong, "Time-dependent intrinsic correlation analysis of crude oil and the US dollar based on CEEMDAN," International Journal of Finance \& Economics, vol. 26, no. 1, pp. 834-848, 2021.

[63] Y. Liu, L. Wang, L. Yang, X. Liu, and L. Wang, "Runoff prediction and analysis based on improved CEEMDAN-OSQR-ELM," IEEE Access, vol. 9, p. 1, 2021.

[64] Z. Wu and N. E. Huang, "Ensemble empirical mode decomposition: a noise-assisted data analysis method," Advances in Adaptive Data Analysis, vol. 1, no. 1, pp. 1-41, 2009.

[65] P. Flandrin, G. Rilling, and P. Goncalves, "Empirical mode decomposition as a filter bank," IEEE Signal Processing Letters, vol. 11, no. 2, pp. 112-114, 2004.

[66] R. J. Gledhill, Methods for Investigating Conformational Change in Biomolecular Simulations, A Dissertation for the Degree of Doctor of Philosophy at the Department of Chemistry, University of Southampton, Southampton, UK, 2003.

[67] R. V. L. Hartley, "Transmission of Information1," Bell System Technical Journal, vol. 7, no. 3, pp. 535-563, 1928.

[68] S. Behrendt, T. Dimpfl, F. J. Peter, and D. J. Zimmermann, "RTransferEntropy-quantifying information flow between different time series using effective transfer entropy," Software, vol. 10, Article ID 100265, 2019.

[69] T. Dimpfl and F. J. Peter, "Using transfer entropy to measure information flows between financial markets," Studies in Nonlinear Dynamics and Econometrics, vol. 17, no. 1, pp. 85-102, 2013.

[70] C. E. Shannon, "A mathematical theory of communication," Bell System Technical Journal, vol. 27, no. 3, pp. 379-423, 1948.

[71] S. Kullback and R. A. Leibler, "On information and sufficiency," The Annals of Mathematical Statistics, vol. 22, no. 1, pp. 79-86, 1951.

[72] C. Beck and F. Schögl, Thermodynamics of Chaotic Systems: An Introduction, Cambridge University Press, Cambridge, UK, 1993.

[73] R. Marschinski and H. Kantz, "Analysing the information flow between financial time series," The European Physical Journal B, vol. 30, no. 2, pp. 275-281, 2002.

[74] S. J. Salyer, J. Maeda, S. Sembuche et al., "The first and second waves of the COVID-19 pandemic in Africa: a cross-sectional study," The Lancet, vol. 397, no. 10281, pp. 1265-1275, 2021.

[75] E. J. d. A. L. Pereira, P. J. S. Ferreira, M. F. da Silva, J. G. V. Miranda, and H. B. B. Pereira, "Multiscale network for 20 stock markets using DCCA," Physica A: Statistical Mechanics and its Applications, vol. 529, 2019.

[76] K. J. Forbes and F. E. Warnock, "Capital flow waves: surges, stops, flight, and retrenchment," Journal of International Economics, vol. 88, no. 2, pp. 235-251, 2012.

[77] X. Zhao, Y. Sun, X. Li, and P. Shang, "Multiscale transfer entropy: measuring information transfer on multiple time scales," Communications in Nonlinear Science and Numerical Simulation, vol. 62, pp. 202-212, 2018.

[78] J. Bouoiyour, R. Selmi, and M. E. Wohar, "Safe havens in the face of Presidential election uncertainty: a comparison between Bitcoin, oil and precious metals," Applied Economics, vol. 51, no. 57, pp. 6076-6088, 2019. 
[79] J. Bouoiyour, R. Selmi, A. K. Tiwari, and O. R. Olayeni, "What drives Bitcoin price," Economic Bulletin, vol. 36, no. 2, pp. 843-850, 2016.

[80] K. Chahuán-Jiménez, R. Rubilar, H. de la Fuente-Mella, and V. Leiva, "Breakpoint Analysis for the COVID-19 pandemic and its effect on the stock markets," Entropy, vol. 23, no. 1, p. 100, 2021.

[81] O. B. Adekoya and J. A. Oliyide, "How COVID-19 drives connectedness among commodity and financial markets: evidence from TVP-VAR and causality-in-quantiles techniques," Resources Policy, vol. 70, 2021.

[82] B. B. Ahundjanov, S. B. Akhundjanov, and B. B. Okhunjanov, "Information search and financial markets under COVID19," Entropy, vol. 22, no. 7, p. 791.

[83] M. Andersen, "Early evidence on social distancing in response to COVID-19 in the United States," SSRN Electronic Journal, 2020.

[84] E. Bouri, R. Demirer, D. Gabauer, and R. Gupta, "Financial market connectedness: the role of investors' happiness," Finance Research Letters, Article ID 102075, 2021.

[85] H. Yu, W. Chu, Y. Ding, and X. Zhao, "Risk contagion of global stock markets under COVID-19: A network connectedness method," Accounting and Finance, Article ID 12775, 2021.

[86] N. T. Vo and N. T. Hung, "Directional spillover effects and time-frequency nexus between oil, gold and stock markets: evidence from pre and during COVID-19 outbreak," International Review of Financial Analysis, vol. 76, Article ID 101730, 2021. 